\title{
Development and validation of minisatellite markers for Carica papaya
}

\author{
G.A.F. OLIVEIRA ${ }^{1}$, J.L.L. DANTAS ${ }^{2}$, and E.J. OLIVEIRA ${ }^{2 *}$ \\ Universidade Federal do Recôncavo da Bahia, Campus Universitário, Cruz das Almas, 44380-000, BA, Brazil ${ }^{1}$ \\ Embrapa Mandioca e Fruticultura, Cruz das Almas, 44380-000, BA, Brazil ${ }^{2}$
}

\begin{abstract}
Whereas microsatellite markers are well described, there are few studies investigating minisatellites. Therefore, this study aimed to identify, characterize, and validate minisatellite loci for papaya (Carica papaya L.). The entire papaya genome, which covers $330 \mathrm{Mb}$, was used to mine minisatellites with motifs ranging from 6 to $500 \mathrm{bp}$, and at least six replicates and 82 loci were validated in a set of 24 accessions. A total of 1730 minisatellite loci were identified, located in 695 sequences, with an average of one minisatellite every $156 \mathrm{~kb}$. Variation in GC content ranged from 0.00 to $83.84 \%$ with an average of $28.84 \%$ indicating that these papaya minisatellites are AT rich. Motifs of up to 20 bases represented $71.45 \%$ of the markers. In addition, the observed variation in the number of motif repeats was from 6 to 186 with an average of 9.27 per minisatellite. Independent of the classification, the frequency of minisatellites decreased with an increase in the number of repeating units. Among the validated loci, $48.78 \%$ were found to be polymorphic, and the number of alleles $\left(\mathrm{N}_{\mathrm{A}}\right)$ ranged from two to seven with a mean of 3.10. The average polymorphic information content (PIC), expected heterozygosity (He), and observed heterozygosity (Ho) were 0.38, 0.43, and 0.11, respectively. According to genetic diversity parameters, such as NA, He, and PIC, no significant correlations were found among the size of motifs, the number of repeats, and the GC content of these minisatellites. This study clearly demonstrates the polymorphism of minisatellites and their potential use in detection of intraspecific genetic variations in C. papaya. The results will be useful when managing genetic resources and plant breeding.
\end{abstract}

Additional key words: genetic diversity, papaya, polymerase chain reaction, polymorphism.

\section{Introduction}

Papaya (Carica papaya L.) is important to the economy of Brazil which is considered one of the world's largest producers of this crop. According to Ming et al. (2008), papaya represents a model crop for genome analysis due to its diploid nature $(2 \mathrm{n}=18)$ and small-sized genome $(372 \mathrm{Mb})$. Recent findings show that a common ancestor of Caricaceae was distributed in Africa and apparently dispersed to Central America ca. 35 Ma ago. Moreover, the family expanded from Central to South America reaching southern South America during the Late Oligocene/Early Miocene (Carvalho and Renner 2012). However, it is currently cultivated throughout several tropical areas worldwide.

To develop new papaya cultivars, it is necessary to invest in the characterization and evaluation of genetic resources with the aim of introducing additional genetic variability into commercial plantations. Genetic diversity

studies in papaya have been performed using dominant and codominant markers such as random amplified polymorphic DNA (RAPD; Stiles et al. 1993), amplified fragment length polymorphisms (AFLPs; Kim et al. 2002, Oliveira et al. 2011), and simple sequence repeats (SSRs; Ocampo et al. 2007, Oliveira et al. 2010, Matos et al. 2013). In general, codominant markers exhibit a higher ability to reveal polymorphisms when compared with other types of DNA markers (Ocampo et al. 2007). However, papaya presents a very narrow genetic base (Kim et al. 2002), which together with a low capacity to generate molecular information using current technology results in underutilization of these genomic tools for conservation and germplasm use. Therefore, it is necessary to invest efforts in different types of molecular markers, such as minisatellite ones, with a high capacity to reveal polymorphisms.

Submitted 16 March 2015, last revision 16 May 2015, accepted 20 May 2015.

Abbreviations: AFLP - amplified fragment length polymorphisms; He - expected heterozygosity; Ho - observed heterozygosity; $\mathrm{N}_{\mathrm{A}}$ - number of alleles; PIC - polymorphic information content; VNTRs - variable-number tandem repeats.

Acknowledgments: The authors thank the Fundação de Amparo à Pesquisa do Estado da Bahia (Fapesb), Coordenação de Aperfeiçoamento de Pessoal de Nível Superior (CAPES), and Conselho Nacional de Desenvolvimento Científico e Tecnológico (CNPq) for the financial assistance and scholarship support.

* Corresponding author; fax: (+55) 75 33128096, e-mail: eder.oliveira@embrapa.br 
Minisatellites, or variable-number tandem repeats (VNTRs), are defined as repeated sequences in tandem (6 - 100 bp). Polymorphisms in these markers are related to variations in the number of fragments and the ability of minisatellites to hybridize to different loci with some degree of similarity within the genome (Vergnaud and Denoeud 2000). Moreover, minisatellites may be separated and visualized through electrophoresis using conventional agarose gels (Bally et al. 2010), thus avoiding the need for sophisticated equipment to separate and reveal fragments. This attribute makes minisatellites a powerful tool for several types of genetic analyses. Nevertheless, the first method developed for detection of minisatellite loci was an extremely laborious technique using the same methodology employed for restriction

\section{Materials and methods}

The genome of a transgenic genotype SunUp (a female plant) was previously sequenced by Ming et al. (2008) and used for mining minisatellite sequences. The annotated genome is available from the US Department of the Energy's Joint Genome Institute (USDOE JGI) at http://www.phytozome.net/papaya.php. The sequences comprise approximately $330 \mathrm{Mb}$ divided into 5809 contigs.

The sequences were obtained in a FASTA format and then analyzed using the tandem repeats finder $T R F$ program (Benson 1999) to search for repeated sequences that characterize minisatellites; alignment parameters two, five, and seven were used for matches, mismatches, and indels, respectively. A minimum alignment score to report repeats was 30 , and a maximum period size was 500. Minisatellite motifs ranging from 6 to 500 bases were analyzed with at least six minisatellite repeats and over $70 \%$ matches.

Eighty-two minisatellites (Table 1 Suppl.) were randomly selected for validation in a set of papaya germplasms. Primers were designed using the software Primer 3 v. 4.0 (Rozen and Skaletsky 2000) with annealing temperatures ranging from 50 to $60{ }^{\circ} \mathrm{C}$, a maximum difference of $3{ }^{\circ} \mathrm{C}$ between pairs of primers, primer lengths of 18 - 22 bases, and a GC content between 40 and $60 \%$. Default values were used for other parameters.

Initially, two papaya genotypes (Calimosa and Sunrise Solo, 'Formosa', and 'Solo' types, respectively) were used to optimize the PCR conditions for each minisatellite locus. Then, 24 randomly selected accessions from the Papaya Germplasm Bank (PGB) at Embrapa Cassava and Fruits were used for characterization of minisatellite polymorphism (Table 2 Suppl.).

Young leaves of papaya accessions were collected, identified, and stored at $-80^{\circ} \mathrm{C}$. The DNA was extracted using the cetyltrimethylammonium bromide (CTAB) protocol described by Doyle and Doyle (1990) with minor adjustments. fragment length polymorphism (RFLP) analysis (Jeffreys et al. 1985). However, the availability of genome sequences allows development of site-specific minisatellites (codominant), as reported for Oryza and Brassica species (Honma et al. 2011) and humans (Denoeud et al. 2003).

The development of minisatellite markers can increase detection of molecular polymorphism within and among papaya accessions, representing an important tool for genetic analysis of the species. Therefore, this study aimed at identifying and characterizing minisatellite markers via genome mining and validating certain primers in specific papaya genotypes to determine their potential use in several types of genetic analyses.

Quantification of DNA was performed after electrophoresis $\left(3 \mathrm{~V} \mathrm{~cm}^{-1}\right)$ by comparing the samples with a series of known concentrations of Lambda DNA (Invitrogen, Carlsbad, CA, USA). Electrophoresis was carried out using $1.0 \%(\mathrm{~m} / \mathrm{v})$ agarose gels with a TBE running buffer $(0.89 \mathrm{M}$ Tris, $\mathrm{pH} 8.0,0.89 \mathrm{M}$ boric acid, $0.02 \mathrm{M}$ EDTA, $\mathrm{pH} 8.1$ ) at a $0.5 \times$ concentration. Content of DNA was estimated through a visual comparison of the intensities of the bands revealed by staining with ethidium bromide $\left(10 \mathrm{mg} \mathrm{cm}^{-3}\right)$. Subsequently, the DNA content was standardized to $2.5 \mu \mathrm{g} \mathrm{dm}{ }^{-3}$.

For minisatellite optimization, PCR assays were performed using genotypes Calimosa and Sunrise Solo in a final volume of $15 \mathrm{~mm}^{3}$ containing $10 \mathrm{ng}$ of DNA, $0.2 \mathrm{mM}$ each primer, $0.2 \mathrm{mM} \mathrm{dNTPs}$, and $0.5 \mathrm{U}$ of Taq DNA polymerase (Ludwig Biotec, Alvorada, Brazil), employing different temperatures and $\mathrm{MgCl}_{2}$ concentrations. Amplifications under different conditions were performed in a Veriti ${ }^{\circledR}$ 96-Well thermocycler (Applied Biosystems, Foster City, CA, USA), and the products were separated by electrophoresis on a $2 \%$ $(\mathrm{m} / \mathrm{v})$ agarose gel (Invitrogen) which was stained with ethidium bromide $\left(10 \mathrm{mg} \mathrm{cm}^{-3}\right)$ and visualized under UV radiation.

After determining the best PCR conditions, 24 accessions from the PGB were genotyped to determine the potential use of minisatellites for polymorphism detection. The amplification products were separated by electrophoresis on $3 \%(\mathrm{~m} / \mathrm{v})$ agarose gels (Invitrogen), stained with ethidium bromide $\left(10 \mathrm{mg} \mathrm{cm}^{-3}\right)$, and viewed with a Carestream Gel Logic 212 Pro (Kodak, Rochester, NY, USA). The molecular mass of the polymorphic loci was determined based on comparison with a standard 50 bp molecular mass ladder (Ludwig Biotec).

A descriptive analysis of minisatellite loci was performed by estimating a number of alleles per locus, observed heterozygosity (Ho), expected heterozygosity (He), and polymorphic information content (PIC) according to Botstein et al. (1980). 


\section{Results and discussion}

Through analysis of 5809 contigs and supercontigs covering approximately $330 \mathrm{Mb}(88.71 \%$ of the papaya genome), a total of 1730 microsatellite loci in 146 contigs and 549 supercontigs were identified. The minisatellite loci were located in sequences covering $271 \mathrm{Mb}(72.85 \%$ coverage of the papaya genome according to Ming et al. 2008). Therefore, we identified on average one minisatellite every $156 \mathrm{~kb}$. Although there are no current reports on the development of minisatellites in papaya, it is possible to establish some comparisons with other tandem repeats. For example, Eustice et al. (2008) analyzed $35.5 \mathrm{Mb}$ of the papaya genome and identified one microsatellite every $37.8 \mathrm{~kb}$. The differences in the frequencies of these repeats in the papaya genome may be due to variations in the search criteria and algorithms used for their detection and due to differences in the type and size of the data sets employed as well as due to the inbreeding (or domestication) level of each genotype. In other species, microsatellites have been found to be more frequent. For example, Cavagnaro et al. (2010) analyzed approximately $55.31 \%$ of the Cucumis sativus genome and found one microsatellite every $1.8 \mathrm{~kb}$. Considering their greater size and lower mutation rate, minisatellites appear to be more widely spaced in the papaya genome than microsatellites.

Most of the obtained contigs $(88.36 \%)$ and supercontigs $(56.36 \%)$ exhibited only one minisatellite sequence. Only 17 contigs showed more than 2 minisatellites, and 4 minisatellites were found in only 2 contigs. In contrast, several supercontigs presented more than 2 minisatellites, some of which (5.84 \%) exhibited more than 10 minisatellites (Fig. 1), most likely because of their size and better sequence organization.

Due to a greater complexity of mutation processes associated with minisatellites, the internal conservation of motifs used as predictors of microsatellite polymorphisms cannot be applied to minisatellites (Denoeud et al. 2003). Therefore, considering the difficulty in classifying minisatellites into specific motifs, as it is performed for microsatellites, and taking into account a specific process of mutation and generation of new alleles, which promotes the major interruption of minisatellite loci, we adopted the GC content as criterion for classification of these markers (Fig. 2).

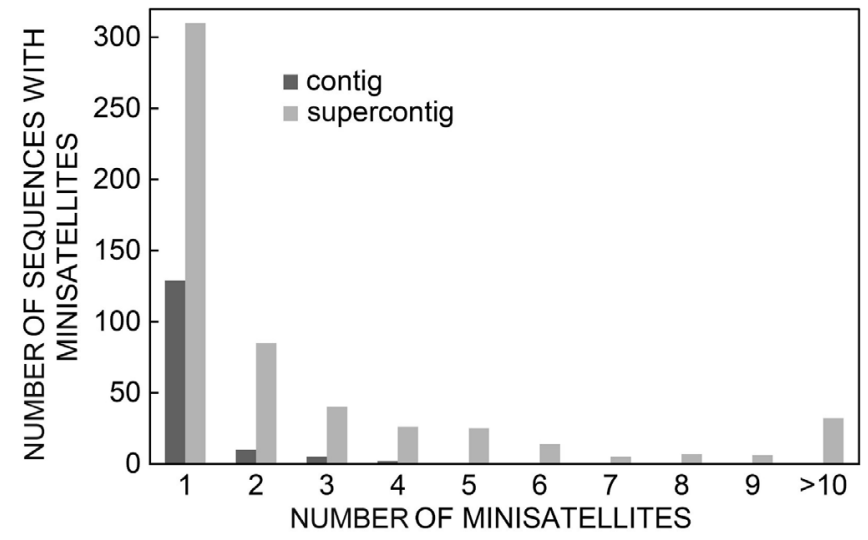

Fig. 1. The distribution of minisatellite sequences in different contigs and supercontigs of papaya genome sequences.

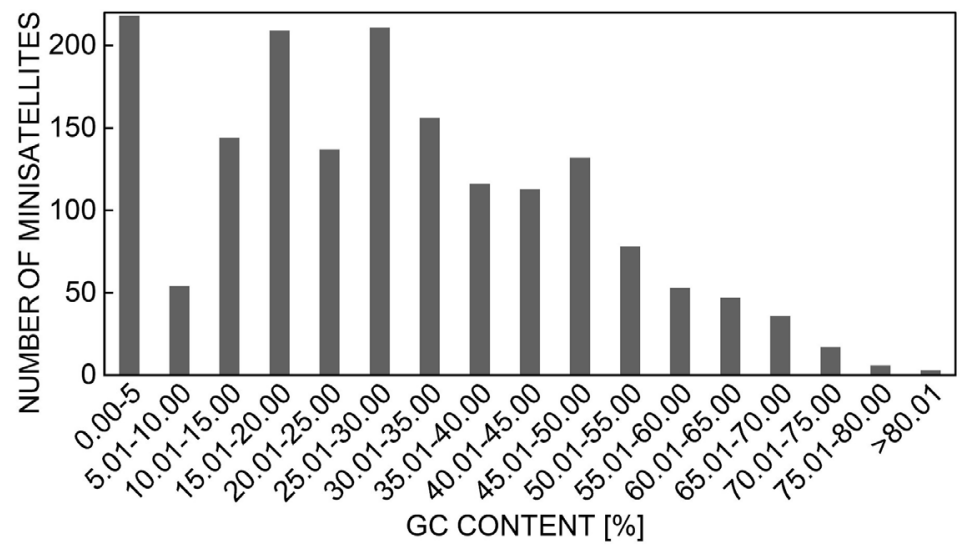

Fig. 2. The distribution of the GC content of minisatellite motifs identified in the papaya genome. 
In general, the observed GC content ranged from 0.00 to $83.84 \%$, with an average of $28.84 \%$, indicating that these minisatellites were rich in the $\mathrm{A}$ and $\mathrm{T}$ nucleotides. The number of minisatellites with a GC content above $50 \%$ was quite intermediate with only three loci showing a GC content over $80 \%$ (Fig. 2). Similar results were observed by Zakrzewski et al. (2010), who reported identification and characterization of repetitive sequences in the Beta vulgaris genome with nine identified minisatellite families showing a GC content ranging between 24 and $33 \%$. By analyzing $35.5 \mathrm{Mb}(9.54 \%)$ of the papaya genome, Eustice et al. (2008) found that AT-rich repeats are predominant regardless of the motif size of microsatellites. As example, AT/TA repeats were found to account for $69.20 \%$ of the observed dinucleotides with AAG/TTC and ATT/TTA repeats representing more than $84 \%$ of the trinucleotides.
Regarding the motif size of the minisatellites, 30.00, 8.03 , and $3.76 \%$ of the markers were classified as hexa-, hepta- and octa-nucleotides, respectively (Fig. 3). Motifs of up to 10 bases represented $55.95 \%$ of the minisatellites, and those of up to 20 bases included most of the minisatellites $(71.45 \%)$. Motifs greater than 100 bases accounted for only $9.25 \%$ of the minisatellites. In general, the representation of motif size among tandem repeats was highly variable and depends on the species. Of 84 minisatellites identified in Brassica and Oryza, most of the motifs present range from 20 to $72 \mathrm{bp}$, whereas 67 minisatellites exhibit motifs between 22 and 62 bp (Honma et al. 2011). Searching for microsatellite sequences, Eustice et al. (2008) found that dinucleotide repeats are most frequent in the papaya genome $(74.20 \%)$, followed by trinucleotides $(18.87 \%)$, tetranucleotides $(5.86 \%)$, pentanucleotides $(0.75 \%)$, and hexanucleotides $(0.32 \%)$.

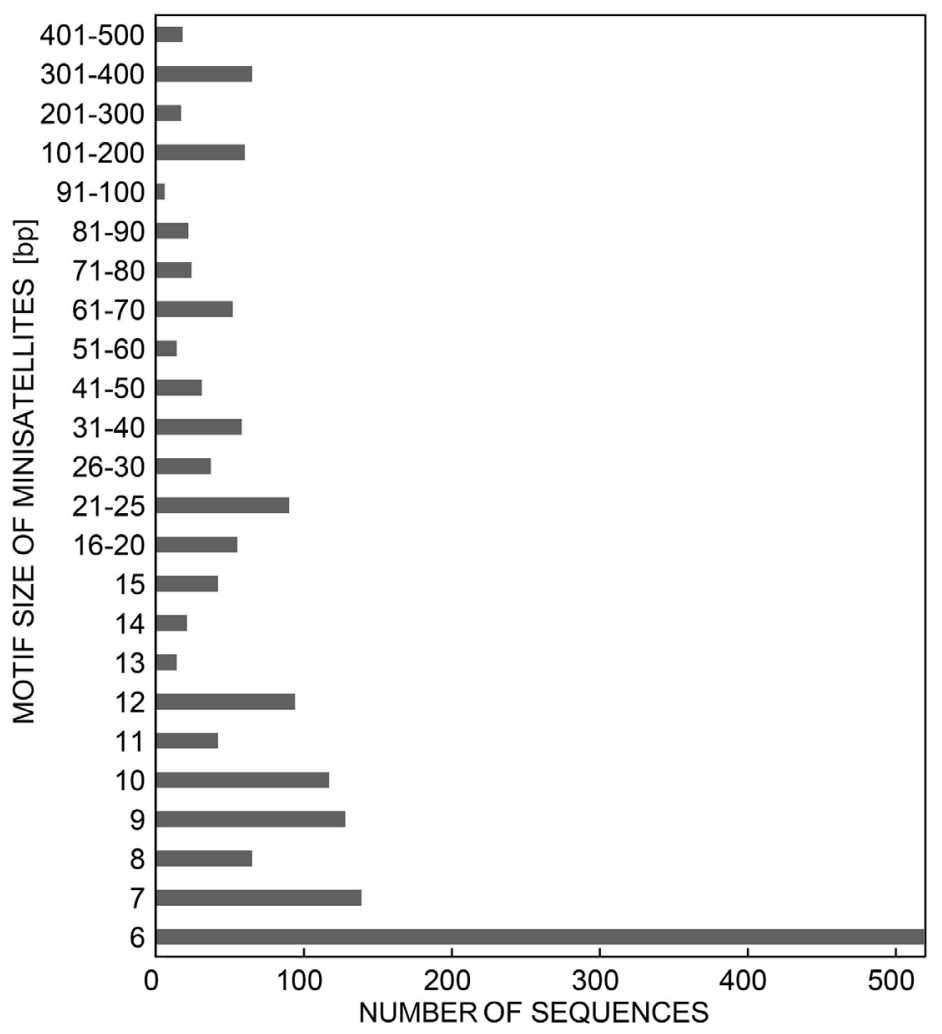

Fig. 3. The distribution of sequences according to the motif size of minisatellites in the papaya genome.

Regarding a number of minisatellite motifs, the observed range was 6 - 186 repeats with an average of 9.27 repeats. In relative terms, $37.7 \%$ of the loci showed between 6.0 and 7.0 repeats, $20.7 \%$ between 7.1 and 8.0 repeats, and $12.5 \%$ between 8.1 and 9.0 repeats. Therefore, motifs of up to nine repeats represented more than $70 \%$ of the observed minisatellites (Fig. 4). Regardless of the classification, the frequency of microsatellites decreased with an increasing number of repeats. However, this change was more gradual for smaller motifs (mainly motifs of six and seven nucleotides) for which greater numbers of motif repeats were observed. Similar observations were made for microsatellites for which the number of dinucleotide repeats (9.6) was found to be nearly twice the number of trinucleotide repeat units (4.9) and three times greater than that of tetra- to octa-nucleotides (3.2 to 3.3 ) (Cavagnaro et al. 2010). In general, sequences with many repeats tend to accumulate mutations and recombination events that limit their expansion generating base exchange that leads to imperfections in motifs and hence to mischaracterization of these loci. 
The PCR optimization of 82 minisatellites was performed using two papaya genotypes (Calimosa and Sunrise Solo). Sixteen minisatellite loci (CPMini-05, CPMini-06, CPMini-11, CPMini-17, CPMini-18, CPMini-20, CPMini-23, CPMini-33, CPMini-34, CPMini-54, CPMini-67, CPMini-68, CPMini-73, CPMini-74, CPMini-81, and CPMini-84) showed either no amplification product or a smear on the gel and were therefore discarded from further analysis. For the remaining 66 minisatellite loci, the PCR amplification conditions were adjusted for specific expected sizes of the fragments according to the primer design. The main adjustment made to the PCR conditions was the $\mathrm{MgCl}_{2}$ concentration, with the concentrations for 38 and 27 loci being adjusted to $2.0 \mathrm{mM}$ and $1.5 \mathrm{mM}$, respectively (Table 3 Suppl.). Regarding the amplification program, annealing temperatures for the minisatellite loci were adjusted to a range from 45 to $64{ }^{\circ} \mathrm{C}$, though a half of these loci did show a good pattern of amplification using annealing temperatures ranging from 58 to $60^{\circ} \mathrm{C}$.

Sixty-six minisatellites were used for analysis of polymorphisms in a set of 24 papaya accessions. Of these minisatellites, 26 were found to be monomorphic and were therefore excluded from the analyses. The rate of polymorphic minisatellites in papaya $(48.78 \%)$ is comparable to that reported by other authors for validation of microsatellite markers in cucumber (48.31\% - Cavagnaro et al. 2010) and papaya (52.88\% Eustice et al. 2008 and $59.00 \%$ - Oliveira et al. 2010) but lower than $75.00 \%$ of polymorphic minisatellites observed in humans (Denoeud et al. 2003).

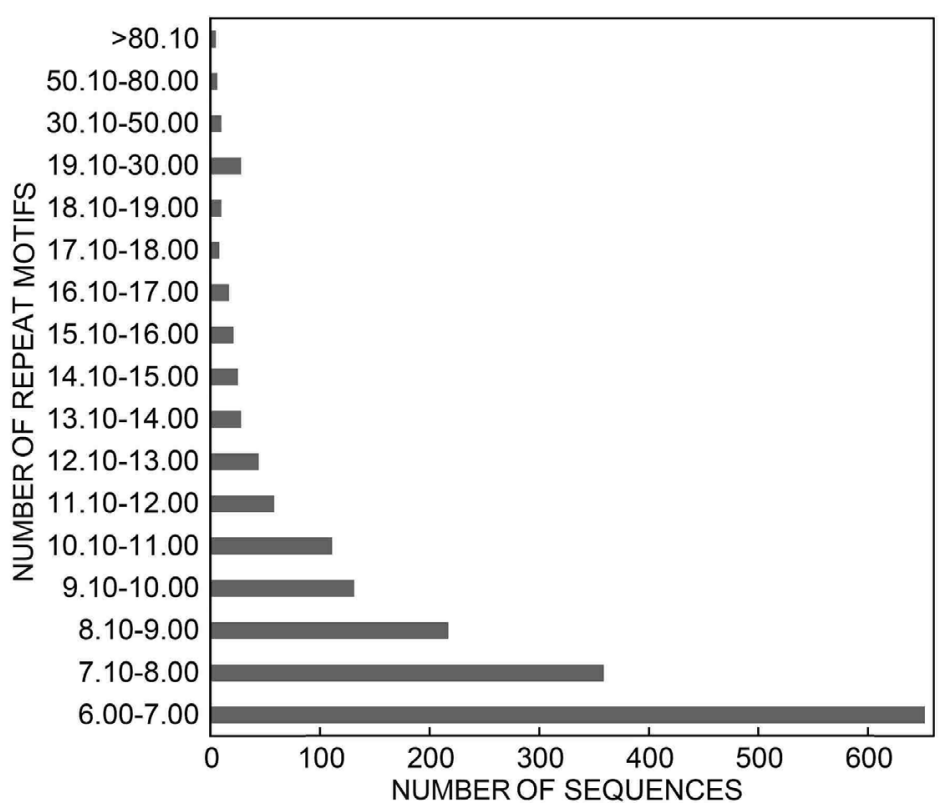

Fig. 4. The distribution of sequences according to the number of motif repeats in minisatellites identified in the papaya genome.

For the remaining 40 polymorphic minisatellites (Table 1), a number of alleles ranged from 2 to 7 ; loci with the largest number of alleles were CPMini-16 (7 alleles), CPMini-01 and CPMini-04 (6 alleles), and CPMini-21 (5 alleles). An average number of minisatellite alleles was 3.10. In Leptosphaeria maculans L., analysis of seven polymorphic minisatellites revealed an average of 5.00 alleles per locus (Jędryczka et al. 2010). As there are no minisatellites developed for papaya to date, microsatellites should be utilized for comparisons. Some reports have shown that in comparison with minisatellites, microsatellites present a greater number of alleles per locus. For example, using 59 markers, Oliveira et al. (2010) identified 4.02 alleles per locus in analysis of 30 germplasm accessions and 18 papaya cultivars. Additionally, Ocampo et al. (2006b) found an average of 3.82 alleles in evaluation of 29 accessions of $C$. papaya and 11 accessions of Vasconcellea spp. Moreover, Ocampo et al. (2007) reported an average of 6.60 alleles per microsatellite when evaluating 72 papaya accessions, and Matos et al. (2013) reported a range of 3 to 10 alleles with an average of 4.53 alleles per locus when evaluating a wide range of papaya accessions (96).

A smaller number of minisatellite alleles may be related to a reduced number of genotypes, a high inbreeding of accessions, a low variability inherent to these approaches, or even to a natural process of minisatellite generation, which leads to a reduced number of new minisatellite alleles compared with microsatellites (Ellegren 2004).

Several studies have shown a low molecular variation within C. papaya based on the AFLP (Kim et al. 2002, Oliveira et al. 2011) and microsatellite markers (Oliveira et al. 2010). These findings have encouraged efforts in developing new markers that are more polymorphic or allow automation of genotyping with a high accuracy in molecular analyses. Among these new molecular 
markers, minisatellites show a high potential to be employed as reference genomic tool because fewer minisatellite alleles per locus may be advantageous for establishing multiplex genotyping systems, as minor variations in allele size allow the use of a larger number of fluorescence dyes in the same spectrum (Faria et al. 2011). Loci with larger motifs have been used in refined analyses that require a high accuracy such as genetic identity and paternity analyses in humans (Ruitberg et al. 2001).

In our study, the size of the amplicons varied from $130-975 \mathrm{bp}$ as expected due to the primer design. The PIC values ranged from 0.04 (CPMini-22) to 0.73
(CPMini-16) with a mean of 0.39. According to the classification of Botstein et al. (1980), PIC values can be grouped into three levels: highly informative (PIC $>0.5)$; moderately informative $(0.25<\mathrm{PIC}<0.50)$, and uninformative (PIC $<0.25$ ). Thus, 25, 50, and $25 \%$ of the papaya minisatellite markers could be considered highly informative, moderately informative, and uninformative, respectively. On average, the PIC values of these minisatellites can be considered moderately informative (0.39).

The estimates of He varied widely among the loci showing a range from 0.04 (CPMini-22) to 0.76 (CPMini-16), with an average of 0.43 ; the Ho estimates

Table 1. The number of alleles per locus $\left(\mathrm{N}_{\mathrm{A}}\right)$, number of genotypes $\left(\mathrm{N}_{\mathrm{G}}\right)$, size range of fragments, expected heterozygosity (He), observed heterozygosity (Ho), and polymorphism information content (PIC) in minisatellite loci in papaya accessions.

\begin{tabular}{|c|c|c|c|c|c|c|}
\hline Loci & $\mathrm{N}_{\mathrm{A}}$ & $\mathrm{N}_{\mathrm{G}}$ & Range [bp] & $\mathrm{He}$ & Но & PIC \\
\hline CPMini-01 & 4 & 6 & $340-400$ & 0.56 & 0.25 & 0.49 \\
\hline CPMini-03 & 2 & 1 & $350-650$ & 0.50 & 1.00 & 0.38 \\
\hline CPMini-04 & 6 & 8 & $330-500$ & 0.75 & 0.29 & 0.71 \\
\hline CPMini-07 & 2 & 2 & $950-960$ & 0.31 & 0.00 & 0.26 \\
\hline CPMini-08 & 3 & 3 & $290-310$ & 0.34 & 0.08 & 0.31 \\
\hline CPMini-09 & 3 & 3 & $285-300$ & 0.16 & 0.08 & 0.15 \\
\hline CPMini-10 & 3 & 3 & $960-975$ & 0.67 & 0.00 & 0.59 \\
\hline CPMini-14 & 3 & 3 & $745-755$ & 0.32 & 0.00 & 0.29 \\
\hline CPMini-15 & 4 & 5 & $480-500$ & 0.46 & 0.13 & 0.42 \\
\hline CPMini-16 & 7 & 8 & $130-170$ & 0.76 & 0.25 & 0.73 \\
\hline CPMini-19 & 2 & 2 & $450-460$ & 0.41 & 0.00 & 0.32 \\
\hline CPMini-21 & 5 & 9 & $350-420$ & 0.68 & 0.38 & 0.62 \\
\hline CPMini-22 & 2 & 2 & $400-410$ & 0.04 & 0.04 & 0.04 \\
\hline CPMini-24 & 2 & 3 & $160-180$ & 0.49 & 0.04 & 0.37 \\
\hline CPMini-25 & 2 & 2 & $220-230$ & 0.28 & 0.00 & 0.24 \\
\hline CPMini-27 & 3 & 4 & $330-390$ & 0.48 & 0.17 & 0.40 \\
\hline CPMini-28 & 3 & 3 & $280-310$ & 0.19 & 0.13 & 0.18 \\
\hline CPMini-30 & 3 & 3 & $450-475$ & 0.35 & 0.00 & 0.32 \\
\hline CPMini-31 & 3 & 3 & $285-305$ & 0.35 & 0.17 & 0.32 \\
\hline CPMini-36 & 2 & 2 & $390-400$ & 0.49 & 0.00 & 0.37 \\
\hline CPMini-37 & 4 & 6 & $345-370$ & 0.66 & 0.33 & 0.60 \\
\hline CPMini-39 & 3 & 5 & $280-300$ & 0.62 & 0.25 & 0.54 \\
\hline CPMini-40 & 3 & 4 & $390-410$ & 0.49 & 0.13 & 0.43 \\
\hline CPMini-43 & 2 & 2 & $780-790$ & 0.26 & 0.00 & 0.22 \\
\hline CPMini-44 & 3 & 3 & $380-390$ & 0.60 & 0.00 & 0.53 \\
\hline CPMini-46 & 5 & 5 & $320-355$ & 0.42 & 0.04 & 0.39 \\
\hline CPMini-47 & 3 & 4 & $400-420$ & 0.66 & 0.41 & 0.58 \\
\hline CPMini-48 & 4 & 6 & $320-350$ & 0.65 & 0.13 & 0.58 \\
\hline CPMini-49 & 2 & 2 & $310-320$ & 0.22 & 0.00 & 0.19 \\
\hline CPMini-56 & 2 & 2 & $250-265$ & 0.08 & 0.00 & 0.08 \\
\hline CPMini-62 & 3 & 3 & $240-260$ & 0.45 & 0.00 & 0.41 \\
\hline CPMini-63 & 2 & 2 & $440-450$ & 0.16 & 0.00 & 0.15 \\
\hline CPMini-64 & 3 & 3 & $820-850$ & 0.23 & 0.00 & 0.21 \\
\hline CPMini-65 & 2 & 3 & $170-190$ & 0.38 & 0.17 & 0.30 \\
\hline CPMini-69 & 3 & 3 & $220-240$ & 0.30 & 0.00 & 0.27 \\
\hline CPMini-72 & 2 & 2 & $200-210$ & 0.09 & 0.00 & 0.08 \\
\hline CPMini-75 & 2 & 2 & $460-470$ & 0.35 & 0.00 & 0.29 \\
\hline CPMini-76 & 4 & 5 & $170-185$ & 0.74 & 0.04 & 0.69 \\
\hline CPMini-82 & 4 & 5 & $200-220$ & 0.53 & 0.04 & 0.47 \\
\hline CPMini-83 & 4 & 5 & $200-220$ & 0.53 & 0.04 & 0.47 \\
\hline Average & 3.10 & 3.68 & & 0.42 & 0.11 & 0.38 \\
\hline
\end{tabular}


ranged from 0.00 (CPMini-56, CPMini-72, CPMini-63, CPMini-49, CPMini-64, CPMini-43, CPMini-25, CPMini-69, CPMini-07, CPMini-14, CPMini-30, CPMini-75, CPMini-19, CPMini-62, CPMini-36, CPMini-44, and CPMini-10) to 1.00 (CPMini-03) with a mean of 0.11 (Table 1). This wide variation in the Ho and He values is result of the range of the number of alleles per locus and their frequency distribution. The finding of Ho $=0.0$ for 17 minisatellite loci can be explained by a low number of alleles at these loci (2 - 3 alleles) and their homozygous state.

Using 81 microsatellite markers in analysis of 30 germplasm accessions and 18 local papaya cultivars, Oliveira et al. (2010) identified Ho values ranging from 0.00 to 0.85 and $\mathrm{He}$ values from 0.08 to 0.82 with averages of 0.19 and 0.59 , respectively. In comparison with microsatellites, the lower Ho and He estimates for minisatellites may due to inherent characteristics, the genetic population used, or even differences in the number and representation of the accessions employed for marker validation.

There was no significant linear correlation found in the minisatellites among the following: 1) the motif size and genetic diversity parameters such as $\mathrm{N}_{\mathrm{A}}\left(R^{2}=0.001\right)$, He $\left(R^{2}=0.013\right)$, and PIC $\left.\left(R^{2}=0.011\right) ; 2\right)$ the number of motif repeats and $\mathrm{N}_{\mathrm{A}}\left(R^{2}=0.027\right)$, He $\left(R^{2}=0.029\right)$, and PIC $\left.\left(R^{2}=0.028\right) ; 3\right)$ the GC content [\%] and $\mathrm{N}_{\mathrm{A}}$ $\left(R^{2}=0.041\right)$, He $\left(R^{2}=0.004\right)$, and PIC $\left(R^{2}=0.000\right)$.

The minisatellites presenting a larger difference in motif size (e.g., 6 bases; CPMini-22, CPMini-49, CPMini31, and CPMini-36) showed a variation in the number of alleles (2 to 3$)$, He (0.0 to 0.49$)$, and PIC (0.04 to 0.37 ), slightly below the values observed for the minisatellites with more than 30 bases (CPMini-63, CPMini-15, CPMini-83, CPMini-64, and CPMini-75), for which the range of genetic diversity parameters was as follows: the number of alleles (2 to 4$)$, He (0.16 to 0.53 ), and PIC (0.15 to 0.47$)$. There was no association among the number of motif repeats and the number of alleles, He, and PIC $\left(R^{2}=0.0273,0.0292\right.$, and 0.0279, respectively). Other studies have also demonstrated a low correlation between different motifs, their numbers and the lengths of microsatellite loci in Ipomoea trifida and molecular polymorphism (Hu et al. 2004).

A clear example of lack of association between polymorphisms and GC content was provided by the loci CPMini-44 and CPMini-39, with the GC content of 0.00 and $67.68 \%$, respectively; nonetheless, the He $(0.60 \times$ $0.62)$ and PIC $(0.53 \times 0.54)$ parameters were very similar for these two markers. Lack of association between the polymorphism and GC content observed in the papaya minisatellites is in disagreement with the results of Vergnaud and Denoeud (2000) in humans where most classical minisatellites (polymorphic and/or hypervariable) are considered to be GC rich, with strong asymmetry being observed between the content of purines and pyrimidines. However, other studies have shown that AT-rich minisatellites can also be hypervariable (Yamauchi et al. 2000).
In view of these observations, genetic diversity parameters (the number of alleles, He, and PIC) should be taken into account in the choice of minisatellite loci for genetic analysis in papaya, independently of the number of repeats, size, and motif composition.

Minisatellites have been widely used in various plants for analyses of evolution (Honma et al. 2011), genetic diversity (Cavagnaro et al. 2010), gene expression (Espley et al. 2009), and genetic mapping (Zakrzewski et al. 2010). In addition to their usefulness as genetic markers, minisatellites play an important role in genome evolution, such as in creation of genetic variability and transcriptional regulation (Espley et al. 2009), and they may therefore be useful not only for understanding evolutionary processes at the molecular level but also for understanding generation and maintenance of agronomic traits of interest.

Despite the advances achieved with the sequencing of the papaya genome (Ming et al. 2008), the number of informative markers presenting a low cost, easy laboratory implementation, and a high accuracy in genotypic identification, such as minisatellites, is still insufficient for use in molecular breeding programs. According to Kim et al. (2002), Ocampo et al. (2006a), and Dias et al. (2011), papaya displays a high phenotypic variation with regard to morphological and agronomic characteristics, including fruit size and shape, pulp colour, flavor and fruit sweetness, duration of the juvenile period, plant height, presence of carpeloidy, and flower abortion. However, despite to its high phenotypic variation, moderate levels of intraspecific polymorphism have been observed in several molecular analyses (Stiles et al. 1993, Kim et al. 2002, Eustice et al. 2008, Oliveira et al. 2010, 2011).

Development and combination of different types of molecular markers with a wide genome coverage, such as minisatellites and microsatellites, could be used for genotyping in papaya breeding programs and diversity germplasm studies, especially for genotype/phenotype association and detection of multiple interactions between alleles and phenotypes. This strategy has gained importance, since most of the traits of interest in papaya breeding are controlled by quantitative trait loci highly dependent on environmental conditions (Blas et al. 2012, Jesus et al. 2013). Quantitative trait loci mapping using molecular markers is a useful tool to unlock the genetic basis of complex phenotypic variation by its capacity to improve breeding efficiency by enabling marker-assisted selection. Therefore, a rapid progress for complex traits in papaya breeding programs is expected by using minisatellite markers as additional molecular tool.

To our knowledge, this is the first attempt to develop and validate minisatellite markers for papaya. This study clearly demonstrates the polymorphism of these markers and their potential use in detection of intraspecific genetic variations in different $C$. papaya accessions. Thus, it is possible to identify 1730 minisatellite loci distributed in the papaya genome (covering $72.8 \%$ of the genome), which may facilitate construction of genetic maps of a 
high resolution essential for applications such as positional gene cloning, comparative and associative

\section{References}

Bally, P., Grandaubert, J., Rouxel, T., Balesdent, M.H.: Fonzie: an optimized pipeline for minisatellite marker discovery and primer design from large sequence data sets. - BMC Res. Notes 3: 322, 2010.

Benson, G.: Tandem Repeats Finder: a program to analyze DNA sequences. - Nucl. Acids Res. 27: 573-580, 1999.

Blas, A.L., Yu, Q., Veatch, O.J., Paull, R.E., Moore, P.H., Ming, R.: Genetic mapping of quantitative trait loci controlling fruit size and shape in papaya. - Mol. Breed. 29: 457-466, 2012.

Botstein, D., White, R.L., Skolnick, M., Davis, R.W.: Construction of a genetic linkage map in man using restriction fragment length polymorphism. - Amer. J. human Genet. 32: 314-331, 1980.

Carvalho, F.A, Renner, S.S.: A dated phylogeny of the papaya family (Caricaceae) reveals the crop's closest relatives and the family's biogeographic history. - Mol. Phylogen. Evol. 65: 46-53, 2012.

Cavagnaro, P.F., Senalik, D.A., Yang, L., Simon, P.W., Harkins, T.T., Kodira, C.D., Huang, S., Weng, Y.: Genomewide characterization of simple sequence repeats in cucumber (Cucumis sativus L.). - BMC Genomics 11: 569, 2010.

Denoeud, F., Vergnaud, G., Benson, G.: Predicting human minisatellite polymorphism. - Genome Res. 13: 856-867, 2003.

Dias, N.L.P., Oliveira, E.J., Dantas, J.L.L.: [Evaluation of papaya genotypes using agronomic descriptors and estimation of genetic parameters]. - Pesq. Agropec. Bras. 46: 1471-1479, 2011. [In Port.]

Doyle, J.J., Doyle, J.L.: Isolation of plant DNA from fresh tissue. - Focus 12: 13-15, 1990

Ellegren, H.: Microsatellites: simple sequences with complex evolution. - Nat. Rev. Genet. 5: 435-445, 2004.

Espley, R.V., Brendolise, C., Chagné, D., Kutty-Amma, S., Green, S., Volz, R., Putterill, J., Schouten, H.J., Gardiner, S.E., Hellens, R.P., Allana, A.C.: Multiple repeats of a promoter segment causes transcription factor autoregulation in red apples. - Plant Cell 21: 168-183, 2009.

Eustice, M., Yu, Q., Lai, C.W., Hou, S., Thimmapuram, J., Liu, L., Alam, M., Moore, P.H., Presting, G.G., Ming R.: Development and application of microsatellite markers for genomic analysis of papaya. - Tree Genet. Genomes 4: 333341, 2008.

Faria, D.A., Mamani, E.M.C., Pappas, G.J., Jr., Grattapaglia, D.: Genotyping systems for Eucalyptus based on tetra-, penta-, and hexa-nucleotide repeat EST microsatellites and their use for individual fingerprinting and assignment tests. Tree Genet. Genomes 7: 63-77. 2011.

Honma, Y., Yoshida, Y., Terachi, T., Toriyama, K., Mikami, T., Kubo, T.: Polymorphic minisatellites in the mitochondrial DNAs of Oryza and Brassica. - Curr. Genet. 57: 261-270, 2011.

Hu, J., Nakatani, M., Lalusin, A.G., Fujimura, T.: New microsatellite markers developed from reported Ipomoea trifida sequences and their application to sweet potato and its related wild species. - Sci. Hort. 102: 375-386, 2004.

Jędryczka, M., Irzykowski, W., Jajor, E., Korbas, M.: Polymorphism of ten new minisatellite markers in mapping, and genomic selection for accelerating development of new papaya cultivars. subpopulations of phytopathogenic fungus Leptosphaeria maculans differing with metconazole treatment. - J. Plant Protect. Res. 50: 103-109, 2010.

Jeffreys, A.J., Wilson, E., Thein, S.L.: Hypervariable minisatellite regions in human DNA. - Nature 314: 67-73, 1985.

Jesus, O.N, Freitas, J.P.X., Dantas, J.L.L., Oliveira, E.J.: Use of morpho-agronomic traits and DNA profiling for classification of genetic diversity in papaya. - Genet. mol. Res. 12: 6646-6663, 2013.

Kim, M., Moore, P., Zee, F, Fitch, M.M.M., Steiger, D., Manshardt, R., Paull, R., Drew, R., Sekioka ,T., Ming, R.: Genetic diversity of Carica papaya as revealed by AFLP markers. - Genome 45: 503-512, 2002.

Matos, E.L.S., Oliveira, E.J., Jesus, O.N., Dantas, J.L.L.: Microsatellite markers of genetic diversity and population structure of Carica papaya. - Ann. appl. Biol. 163: 298-310, 2013.

Ming, R., Hou, S., Feng, Y., Yu, Q., Dionne-Laporte, A., Saw, J.H., Senin, P., Wang, W., Ly, B.V., Lewis, K.L., Salzberg, S.L., Feng, L., Jones, M.R., Skelton, R.L., Murray, J.E., Chen, C., Qian, W., Shen, J., Du, P., Eustice, M., Tong, E., Tang, H., Lyons, E., Paull, R.E., Michael, T.P., Wall, K., Rice, D., Albert, H., Wang, M.L., Zhu, Y.J., Schatz, M., Nagarajan, N., Agbayani, R., Guan, P., Blas, A., Wai, C.M., Ackerman, C.M., Ren, Y., Liu, C., Wang, J., Wang, J., Na, J.K., Shakirov, E.V., Haas, B., Thimmapuram, J., Nelson, D., Wang, X., Bowers, J.E., Gschwend, A.R., Delcher, A.L., Singh, R., Suzuki, J.Y., Tripathi, S., Neupane, K., Wei, H., Irikura, B., Paidi, M., Jiang, N., Zhang, W., Presting, G., Windsor, A., Navajas-Pérez, R., Torres, M.J., Feltus, Alex, F., Porter, B., Li, Y., Burroughs, A.M., Luo, M.C., Liu, L., Christopher, D.A., Mount, S.M., Moore, P.H., Sugimura, T., Jiang, J., Schuler, M.A., Friedman, V., Mitchell-Olds, T., Shippen, D.E., Depamphilis, C.W., Palmer, J.D., Freeling, M., Paterson, A.H., Gonsalves, D., Wang, L., Alam, M.: The draft genome of the transgenic tropical fruit tree papaya (Carica papaya Linnaeus). - Nature 452: 991-996, 2008.

Ocampo, J., d'Eeckenbrugge, G.C., Bruyère, S., Lapeyre de Bellaire, L., Ollitrault, P.: Organization of morphological and genetic diversity of Caribbean and Venezuelan papaya germplasm. - Fruits 61: 25-37, 2006a.

Ocampo, J., d'Eeckenbrugge, G.C, Risterucci, A.M., Dambier, D., Ollitrault, P.: Papaya genetic diversity assessed with microsatellite markers in germplasm from the Caribbean region. - Acta hort. 740: 93-101, 2007.

Ocampo, J., Dambier, D., Ollitrault, P., d'Eeckenbrugge, G.C., Brotier, P., Risterucci, A.M.: Microsatellite DNA in Carica papaya L.: isolation, characterization and transferability to Vasconcellea species. - Mol. ecol. Notes 6: 212-217, $2006 \mathrm{~b}$.

Oliveira, E.J., Amorim. V.B.O., Matos, E.L.S., Costa, J.L., Castellen, M.S., Pádua, J.G., Dantas, J.L.L.: Polymorphism of microsatellite markers in papaya (Carica papaya L.). Plant mol. Biol. Rep. 28: 519-530, 2010.

Oliveira, E.J., Costa, J.L., Santos, L.F., Carvalho, F.M., Silva, A.S., Dantas, J.L.L.: Molecular characterization of papaya genotypes using AFLP markers. - Rev. Bras. Fruticult. 33: 848-858, 2011.

Rozen, S., Skaletsky, H.J.: Primer3 on the www for general 
users and for biologist programmers. - In: Krawetz, S., Misener, S. (ed.): Bioinformatics Methods and Protocols: Methods in Molecular Biology. Pp. 365-386. Human Press, Totowa 2000.

Ruitberg, C.M., Reeder, D.J., Butler, J.M.: STRBase: a short tandem repeat DNA database for the human identity testing community. - Nucl. Acids Res. 29: 320-322, 2001.

Stiles, J.I., Lemme, C., Sondur, S., Morshidi, M.B., Manshardt, R.: Using randomly amplified polymorphic DNA for evaluating genetic relationships among papaya cultivars. Theor. appl. Genet. 85: 697-701, 1993.
Vergnaud, G., Denoeud, F.: Minisatellites.: mutability and genome architecture. - Genome Res. 10: 899-907, 2000.

Yamauchi, M., Tsuji, S., Mita, K., Saito, T., Morimyo, M.: A novel minisatellite repeat expansion identified at FRA16B in a Japanese carrier. - Genes Genet. Syst. 75: 149-154, 2000.

Zakrzewski, F., Wenke, T., Holtgräwe, D., Weisshaar, B., Schmidt, T.: Analysis of a c0t-1 library enables the targeted identification of minisatellite and satellite families in Beta vulgaris. - BMC Plant Biol. 10: 8, 2010. 